\title{
Regression of basal cell carcinomas in multiple sclerosis patient on Aubagio treatment
}

\author{
Judy Park DeWitt, BS ${ }^{1}$; Kleesy L. Thomas, MD²; Ashley Sturgeon, MD²
}

\section{October 5, 2017}

Basal cell carcinoma (BCC) is the most common type of skin cancer. ${ }^{1}$ BCCs can develop on any part of the body. Risk factors include sun exposure, tanning bed use, immunocompromised state, and fair skin. There are various types of BCC, including sclerosing, superficial, pigmented, and nodular. ${ }^{1}$ BCCs are often diagnosed by physical exam, although visualization under microscopy is confirmatory. Treatment options vary depending on the size and location. Excision, Mohs micrographic surgery, cryosurgery, and chemotherapies may be employed. ${ }^{1}$ Here, we report unexpected regression of BCCs in a patient using an oral pharmacologic agent, Aubagio, which is an FDAapproved medication for multiple sclerosis (MS).

A 56-year-old, Caucasian woman with a history of MS presented to clinic with five non-healing, nonitchy, pink, scaly plaques on the lower abdomen for many months. Clinical impression was eczematous dermatitis. Biopsy of the largest lesion revealed a diagnosis of superficial basal cell carcinoma. The patient was scheduled for excision of lesions. At her surgery appointment, 1 month later, the BCCs regressed in size considerably. They were treated with shave removal with subsequent electrodessication and curettage. Patient reported that since the initial visit, the only notable change was the start of Aubagio, a new drug treatment for her MS. A recent 6-month follow-up was negative for $\mathrm{BCC}$ recurrence.

Teriflunomide (Aubagio, Genzyme Corporation, Cambridge, MA) is an FDA-approved disease-modifying

Corresponding author: Kleesy L. Thomas, MD Contact Information: Kleesy.Thomas@ttuhsc.edu DOI: $10.12746 /$ swrccc.v5i21.414 treatment for patients with relapsing multiple sclerosis. MS is a common neurodegenerative disease characterized by loss of oligodendrocytes and demyelination. Currently, there is no cure for MS, but many drugs on the market target the activation of lymphocytes or proliferation of activated lymphocytes. Dihydro-orotate dehydrogenase, a mitochondrial enzyme, has a role in the de novo pyrimidine synthesis pathway, which is important in DNA synthesis. ${ }^{2}$ The proposed mechanism of teriflunomide is the inhibition dihydro-orotate dehydrogenase in a non-competitive and reversible manner. ${ }^{2}$ This pyrimidine synthesis inhibitor drug prohibits the proliferation of activated $T$ and $B$ lymphocytes without causing cell death.

During preclinical research stage, teriflunomide showed CNS lymphocyte infiltration reduction and axonal loss reduction without neurological damage in animals. ${ }^{3}$ In clinical trials, patients taking teriflunomide experienced significantly fewer relapses than the patients taking the placebo. ${ }^{3}$ Teriflunomide is not only used for treatment of MS, but have also been implicated in treatment for melanomas. ${ }^{3}$ Although the use of Aubagio is not a standard treatment of care for BCCs, we report a case of cancer regression after onset of Aubagio treatment in our MS patient.

Keywords: Basal Cell Carcinomas, Multiple Sclerosis, Teriflunomide, Aubagio

From: ${ }^{1}$ School of Medicine, Texas Tech University Health Sciences Center, Lubbock, Texas ${ }^{2}$ Department of Dermatology, Texas Tech University Health Sciences Center, Lubbock, Texas

Submitted: 10/2/2017

Conflicts of interest: none 


\section{REFERENCES}

1. Soyer H, Rigel D, Wurm E. Actinic Keratosis, Basal Cell Carcinoma and Squamous Cell Carcinoma. In: Bolognia J, Jorizzo J, Schaffer J. Dermatology. $3^{\text {rd }}$ ed. Philadelphia: Elsevier Saunders. 2012:108:1773-1793.
2. Bar-Or A, Pachner A, Menguy-Vacheron F, et al. Teriflunomide and its mechanism of action in multiple sclerosis. Drugs 2014;74:659-674.

3. Huang O, Zhang W, Zhi Q, et al. Teriflunomide, an immunomodulatory drug, exerts anticancer activity in triple negative breast cancer cells. Experimental biology and medicine 2015;240:426-437. 